Dimensionality-driven phonon softening and incipient charge density wave instability in $\mathrm{TiS}_{2}$

This content has been downloaded from IOPscience. Please scroll down to see the full text.

2016 EPL 11547001

(http://iopscience.iop.org/0295-5075/115/4/47001)

View the table of contents for this issue, or go to the journal homepage for more

Download details:

IP Address: 134.226.8.83

This content was downloaded on 06/01/2017 at 09:01

Please note that terms and conditions apply.

You may also be interested in:

On the origin of charge-density waves in select layered transition-metal dichalcogenides

K Rossnagel

Suppression and emergence of charge-density waves at the surfaces of layered 1T-TiSe2 and 1T-TaS2

by in situ Rb deposition

K Rossnagel

$\underline{\text { Density functional theory study of phonons in graphene doped with } \mathrm{Li}, \mathrm{Ca} \text { and } \mathrm{Ba}}$

Jelena Peši, Vladimir Damljanovi, Radoš Gaji et al.

First-principles calculations of mechanic and vibration properties of $\mathrm{AgRE}(\mathrm{RE}=\mathrm{Ho}, \mathrm{Er}, \mathrm{Tm})$

intermetallic compounds under pressure

Y Mogulkoc, Y O Ciftci, K Colakoglu et al.

Two-dimensional hexagonal semiconductors beyond grapheme

Bich Ha Nguyen and Van Hieu Nguyen

Prediction of topological insulators in supercubane-like materials based on first-principles

calculations

Guo-Xiang Wang, Shuai Dong and Jing-Min Hou

First-principles studies of the thermodynamic properties of bulk Li

P Staikov, A Kara and T S Rahman

A theoretical review on electronic, magnetic and optical properties of silicene

Suman Chowdhury and Debnarayan Jana 


\title{
Dimensionality-driven phonon softening and incipient charge density wave instability in $\mathrm{TiS}_{2}$
}

\author{
KAPILdeb Dolui $^{(a)}$ and Stefano SAnvito \\ School of Physics, AMBER and CRANN Institute, Trinity College - Dublin 2, Ireland
}

received 25 January 2016; accepted in final form 25 August 2016

published online 15 September 2016

PACS 71.15.Mb - Density functional theory, local density approximation, gradient and other corrections

PACS 71.45.Lr - Charge-density-wave systems

PACS 74.70.Ad - Metals; alloys and binary compounds (including A15, $\mathrm{MgB}_{2}$, etc.)

\begin{abstract}
Density functional theory and density functional perturbation theory are used to investigate the electronic and vibrational properties of $\mathrm{TiS}_{2}$. Within the local density approximation the material is a semimetal both in the bulk and in the monolayer form. Most interestingly we observe a Kohn anomaly in the bulk phonon dispersion, which turns into a charge density wave instability when $\mathrm{TiS}_{2}$ is thinned to less than four monolayers. Such instability, however, disappears when one calculates the electronic structure with a functional, such as the LDA $+U$, which returns an insulating ground state. In this situation charge-doping or strain does not bring back the charge density wave instability, whereas the formation of the TiSSe alloy does.
\end{abstract}

Copyright (c) EPLA, 2016

Introduction. - Titanium disulfide $\left(\mathrm{TiS}_{2}\right)$, a prototypical layered transition metal dichalcogenide (LTMD), consists of covalently bonded $\mathrm{Ti}$ and $\mathrm{S}$ atoms arranged in two-dimensional hexagonal planes (monolayers). In its bulk form the monolayers are stacked together by weak van der Waals forces. Many conflicting results exist in the literature on the electronic structure of bulk $\mathrm{TiS}_{2}$, namely it is still debated whether the material is metallic, semimetallic or semiconducting. Several experimental works report $\mathrm{TiS}_{2}$ to be a semiconductor with a bandgap ranging from 0.05 to $2.5 \mathrm{eV}$. These observations are supported by angle-resolved photo-emission spectra [1], optical absorption [2], Hall [3,4] and transport measurements [5]. At the same time a second set of experiments places $\mathrm{TiS}_{2}$ among metals and semimetals with an indirect bandgap overlap ranging from 0.2 to $1.5 \mathrm{eV}$ (between the $\Gamma$ - and the $L$-point in the Brillouin zone). This experimental evidence is based on extensive resistivity measurements [6], infrared reflectance [7] and X-ray emission and absorption [8]. Such a spread in the experimental results concerning the basic electronic structure of bulk $\mathrm{TiS}_{2}$ may originate from the different growth conditions used, which in turn produce non-stoichiometry samples, through unintentional or intrinsic doping $[9,10]$. Nevertheless, a large

\footnotetext{
(a) Current address: Department of Physics and Astronomy, University of Delaware - Newark, DE 19716-2570, USA; e-mail: doluik@tcd.ie (corresponding author)
}

variation of electronic structure ranging from semimetallic to semiconducting has been reported also in the theory works using ab initio electronic structure calculations [11]. For instance, density functional theory (DFT) within the local density approximation and hybrid functional predict, respectively, a semimetallic [12] and a semiconducting ground state [13].

$\mathrm{TiS}_{2}$ has drawn considerable attention over the past four decades because of the variety of its potential applications. For instance, alkali atoms and organic molecules can be intercalated easily into $\mathrm{TiS}_{2}$ for applications related to both light-weight and high-energy-density batteries $[14,15]$. In particular, it has been demonstrated that for bulk $\mathrm{TiS}_{2}$ the intercalation changes the conductivity [16]. A second interesting aspect is that both the electronic structure and the electron transport properties of $\mathrm{TiS}_{2}$ can be modulated by external pressure. Many experimental $[3,11]$ as well as theoretical $[12,17,18]$ studies describe pressureinduced phase transitions in $\mathrm{TiS}_{2}$. For instance, the transition from a semiconductor to a semimetal phase has been supported by Hall measurements on $\mathrm{TiS}_{2}$ single crystals under a $4 \mathrm{GPa}$ pressure [3]. Such evidence highlights the strong coupling between the electronic properties and the $\mathrm{TiS}_{2}$ structure, a feature shared by many LTMDs.

In fact, the LTMD family in general exhibits strong electron-phonon coupling [14]. Often this results into ground states displaying a macroscopic order. For instance 
bulk $\mathrm{NbSe}_{2}$ and $\mathrm{TaSe}_{2}$ are notoriously known to exist in both a superconducting and a charge density wave (CDW) state [19]. The appearance of the CDW phase can be ascribed to several competing mechanisms such as Peierls instability, exciton insulator instability, Jahn-Teller distortion, or Fermi surface (FS) nesting [19-23]. All the mechanisms are closely related to each other, especially in LTMD materials, and in general they are the result of a competition between the ionic and the electronic system to reduce the total energy. As such, identifying the true cause of the CDW is not a trivial task. For example, in the case of bulk $\mathrm{TiSe}_{2}$, all the mentioned mechanisms for the CDW phase transition have been proposed by different experimental methods [24-26]. Recently a significant experimental effort has been devoted to produce ultra-thin layers of such class of materials either by synthesis [27] or by exfoliation [28]. The appeal of reduced dimension LTMDs is that their electronic and vibrational properties are strongly modified when the thickness reduces down to ultra-thin layers, resulting in further potential for future applications. For example, photo-luminescence emerges due to an indirect to direct bandgap change when the thickness of $\mathrm{MoS}_{2}$ is reduced from bulk to monolayer [29]. In contrast, the $\mathrm{TiSe}_{2}$ phonon spectrum softens with decreasing the layers thickness [30]. A monolayer form of $\mathrm{TiS}_{2}$ has been isolated recently [31,32], however, evidence for the CDW phase has not been reported there yet.

In this work, we study the changes in the electronic and vibrational properties of the $\mathrm{TiS}_{2}$, when the material is thinned from bulk to the monolayer limit. In particular we demonstrate that, if the material is described as metallic, a CDW phase will emerge when the number of layers is reduced below four. However, if one adopts a level of description where the ground state is insulating, such a CDW disappears and cannot be restored at any realistic doping level. Intriguingly, such tendency to structural instability can be altered by strain or alloying, which therefore becomes an interesting tuning factor for the electronic structure.

Methodology. - The electronic and vibrational properties of bulk and monolayer $\mathrm{TiS}_{2}$ have been investigated by using, respectively, ab initio density functional theory $[33,34]$ and density functional perturbation theory [35]. We have considered the local density approximation (LDA) [36] of the exchange and correlation functional, as implemented in the Quantum Espresso package [37], except for a set of calculations (see later) where the LDA $+U$ scheme [38], still within Quantum Espresso, has been employed. A simplified rotational-invariant formulation is adopted to calculate the effective interaction parameter, $U$, in the $\mathrm{LDA}+U$ method, as proposed by Cococcioni and co-worker [39]. The Hubbard $U$ correction is introduced only for the correlated Ti $3 d$ orbitals. Ultra-soft pseudo-potentials describe the core electrons of all the atomic species. The electronic wave function is expanded using plane waves up to a cutoff energy of $70 \mathrm{Ry}$.
Calculations for bulk $\mathrm{TiS}_{2}$ are performed for a periodic structure with the $P \overline{3} m 1$ space group. In contrast, for $\mathrm{TiS}_{2}$ monolayers a $12 \AA$ vacuum region is inserted along the non-periodic direction ( $z$-axis). Geometries are relaxed by conjugate gradient, where both the atomic positions and the cell parameters are allowed to relax until the forces on each atom are less than $0.005 \mathrm{eV} / \AA$. The structural optimization of the undistorted phase is initiated from the symmetric atomic positions, i.e. Ti is at $(0,0,0)$ and the two $\mathrm{S}$ atoms are at $(1 / 3,2 / 3, z)$ and $(2 / 3,1 / 3, z)$ with $z=0.25$ in the bulk unit cell. A total energy tolerance of $10^{-10} \mathrm{Ry}$ is used for each self-consistent cycle during the structural relaxation. The electronic integrations are carried out by using a $16 \times 16 \times 10(20 \times 20 \times 1)$ Monkhorst-Pack $k$-grid for bulk (monolayer) $\mathrm{TiS}_{2}$ and a Hermite Gaussian smearing of $0.01 \mathrm{Ry}$ is used for all the calculations. We have checked that spin-orbit coupling does not have any significant effect on the electronic structures of both the pristine monolayer and bulk $\mathrm{TiS}_{2}$. In computing the phonon spectrum the dynamical matrix is evaluated over a $4 \times 4 \times 2(4 \times 4 \times 1)$ phonon-momentum grid and it is interpolated throughout the Brillouin zone in order to plot the bulk (monolayer) phonon bandstructure.

Results and discussion. - We begin by calculating the electronic properties of bulk $\mathrm{TiS}_{2}$. The LDAoptimized bulk cell parameters are $a=b=3.31 \AA$, $c=$ $5.45 \AA$, in good agreement with the previous theoretical (LDA) values [12] of $a=b=3.312 \AA, c=5.449 \AA$ and with the experimental ones [40] of $a=b=3.407 \AA, c=5.695 \AA$. The Ti-S bond length is found to be $2.38 \AA$, again in close agreement with the experimental value [41] of $2.32 \AA$ and with a previous theoretical (LDA) estimate [18] of $2.383 \AA$. The calculated energy bandstructure along the high symmetry lines in the bulk $\mathrm{TiS}_{2}(\mathrm{P} \overline{3} m 1)$ Brilloiun zone is shown in fig. 1(a). Clearly bulk $\mathrm{TiS}_{2}$ is predicted to be a semimetal with an indirect band-overlap of $0.16 \mathrm{eV}$ (negative bandgap). The valence band maximum is found to be located at the $\Gamma$-point, while the conduction band minimum is at $L$. The corresponding partial density of states (DOS) projected over the different atomic species shows that the valence and the conduction band originate, respectively, from the S $3 p$ and the Ti $3 d$ orbitals, although there is a good degree of hybridization, see fig. 1(b).

Our calculated bandstructure is in good agreement with previous calculations performed at the same level of description [11]. It is important to note, however, that quasi-particle corrections to such bandstructure, as those brought about the $G W$ scheme, return an insulating ground state with a gap slightly smaller than $1 \mathrm{eV}$ [42]. Note, that the actual bandgap is difficult to establish with certainty as it depends sensitively on the fine details of the crystal structure, so that the geometries relaxed with different DFT functionals are associated with different bandgaps.

Next we move to study the electronic properties of $\mathrm{TiS}_{2}$ in its monolayer form (see figs. 1(c) and (d)). Our 

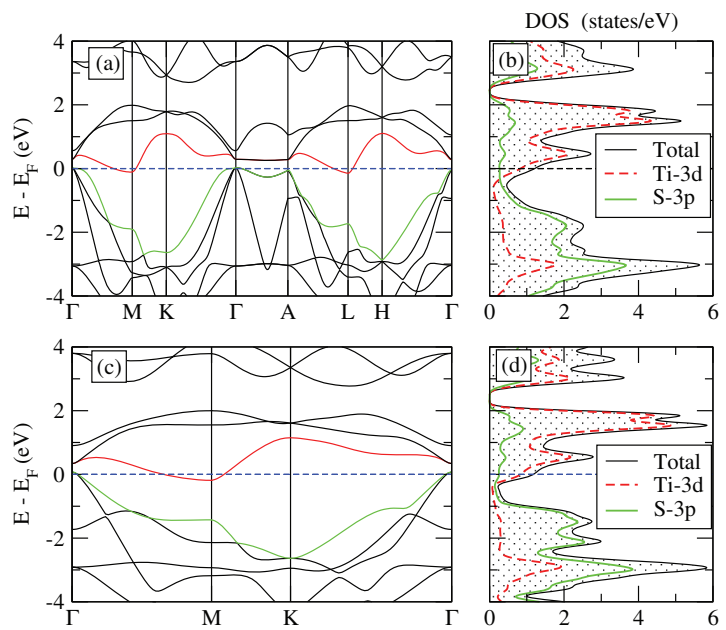

Fig. 1: (Color online) The LDA-bandstructure of bulk (a) and monolayer (c) $\mathrm{TiS}_{2}$. The conduction band (red line) and valence band (green line) overlap between the $\Gamma$ and the $L(M)$ points for bulk (monolayer) $\mathrm{TiS}_{2}$. The density of states (black line) is projected over the $\mathrm{Ti} 3 d$ (red dotted line) and $\mathrm{S} 3 p$ (green line) orbitals for bulk (b) and monolayer (d). The blue dashed line indicates the Fermi level, $E_{\mathrm{F}}$. The electronic states in the DOS are Gaussian broadened by $0.01 \mathrm{Ry}$.

optimized lattice constant, $a=b$, is now $3.32 \AA$. Such value slightly underestimates the experimentally observed one [43] of $3.40 \AA$, as expected from the covalent nature of the Ti-S bond and the general tendency of the LDA to overbind. Our calculations show that the electronic property, i.e. the bandgap, does not change significantly when the thickness decreases from bulk to a single monolayer. Our computed LDA negative bandgap of $-0.25 \mathrm{eV}$ is in good agreement with the previous theoretical calculations at the LDA level [44]. Also for $\mathrm{TiS}_{2}$ monolayers, like in the bulk, the conduction and valence bands are respectively derived from Ti $3 d$ and S $3 p$ orbitals (fig. 1(d)). Again quasi-particle corrections open the bandgap and describe $\mathrm{TiS}_{2}$ monolayers as insulating [42]

We now investigate the effects of the dimensionality on the phonon properties of $\mathrm{TiS}_{2}$ by starting from its bulk form, whose phonon dispersion is plotted in fig. 2(a) along the $\Gamma-M-K-\Gamma-A q$-path. In order to facilitate a comparison with experiments we recall that the irreducible representation of the zone-center phonon modes reads

$$
\Gamma=2 A_{2 u}(\mathrm{IR})+2 E_{u}(\mathrm{IR})+A_{1 g}(\mathrm{R})+E_{g}(\mathrm{R}),
$$

where $\mathrm{R}$ and IR denote, respectively, Raman and infrared active modes. The corresponding atomic displacements of the optical modes at the $\Gamma$-point are shown in fig. 3. Notably the phonon frequencies of a number of modes have been measured experimentally by vibrational spectroscopy, such as Raman, infrared and neutron scattering $[45,46]$. These values are also reported in fig. 3, demonstrating a good agreement with our calculated ones. In addition, our phonon dispersion agrees quite well with
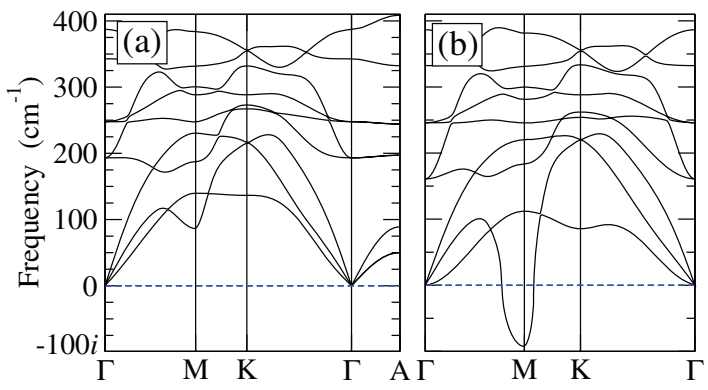

(c)

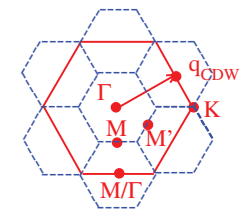

Fig. 2: (Color online) The phonon dispersion of bulk (a) and monolayer (b) $\mathrm{TiS}_{2}$. Note that the calculated imaginary frequencies are shown as negative roots of the square phonon frequencies. Lines are guides to the eye. (c) Brillouin zones of the $(1 \times 1)$ and $(2 \times 2)$ structure outlined in red and blue lines, respectively. $q_{\mathrm{CDW}}$ denotes the $\mathrm{CDW}$ wave vector.

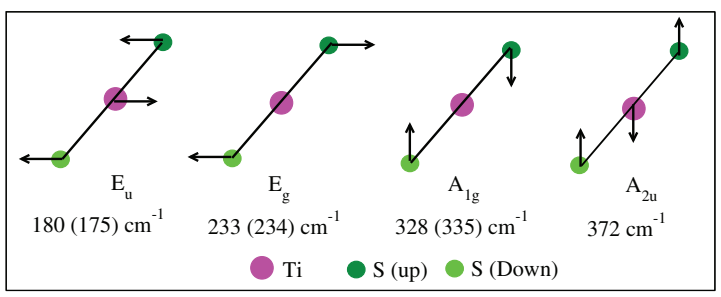

Fig. 3: (Color online) The atomic displacements of the $\Gamma$ centered optical phonon modes of bulk $\mathrm{TiS}_{2}$. Frequencies are reported from our calculations while the corresponding experimental values [45] are in bracket. S (up) and S (down) refer to $\mathrm{S}$ atoms, respectively, on the upper and lower plane relative to the Ti one.

previous theoretical calculations based either on empiricalvalence-force-field methods [45] or on state of the art first-principle density functional perturbation theory [12]. Interestingly, we observe the signature of a soft-phonon mode, generally called Kohn anomaly, at the $M$-point in the Brillouin zone. This signals an incipient structural instability, which is yet not fully realized in the bulk.

The phonon dispersion of $\mathrm{TiS}_{2}$ in the monolayer form is shown in fig. 2(b). Similarly to the bulk phonon dispersion also for the monolayer there is no energy-gap between the optical and the acoustic phonon modes. However, we find that when $\mathrm{TiS}_{2}$ is thinned down to a monolayer the Kohn instability at $M$ becomes a dynamically instability, namely there is now an imaginary phonon frequency at $M$ and the corresponding CDW wave vector is $q_{\mathrm{CDW}}=\frac{\pi}{a}\left(\frac{1}{2}, 0,0\right)$. This reflects the fact that the system gets stabilized by forming either a $1 \times 2$ or a $2 \times 1$ supercell. Consequently, their combined effects yield the formation of a $2 \times 2$ stable supercell (see fig. 2(c)). In 
order to simulate the distorted structures, we initialize the atomic positions by slightly displacing the atoms from their undistorted coordinates and then relax the structure searching for the minimum energy configuration. We find that the system becomes stabilized by forming a $2 \times 2$ superstructure, i.e. a commensurate CDW phase where the atomic positions are distorted. We have also checked that the $2 \times 1$ supercell converges to a distorted phase gaining an energy of about $0.64 \mathrm{meV} /$ formula-unit with respect to the undistorted cell. If one now constructs a $2 \times 2$ supercell such an instability migrates at the $\Gamma$-point, reflecting the fact that $M$ folds to $\Gamma$ in the supercell. Now atomic relaxation of the supercell gives us a new distorted structure, which is $0.85 \mathrm{meV} /$ formula-unit lower in energy than the undistorted one. The phonon bandstructure for such new distorted phase has now only real frequencies, indicating that no further symmetry lowering is present. A schematic diagram of the distorted superstructure is shown in fig. 4(b). The direction of the distortion of the atomic positions are determined by the $q$-vector, at which the unstable phonon mode occurs. In such new CDW phase the displacement of the $\mathrm{Ti}$ atoms, $\delta \zeta_{T i}$ is much larger, $0.05 \AA$, than that of $S$ that is $\leq 0.005 \AA$.

The bandstructure for the monolayer as calculated for the $2 \times 2$ supercell is presented in fig. 4(a) for both the normal and the CDW phase. The crucial aspect is that in the CDW phase the material is able to open a bandgap of $0.09 \mathrm{eV}$, i.e., as expected, the emergence of the CDW phase is associated to a metal-to-insulator transition. The microscopic origin of the CDW phase is mainly associated to the strong electron-phonon coupling in LTMDs, which allows one to modify significantly the bandstructure with moderate lattice distortions. This is a feature common to the entire class of LTMD materials $[47,48]$. A similar CDW phase transition has been observed experientially in $\mathrm{TiSe}_{2}$, a compound iso-structural and isoelectronic to $\mathrm{TiS}_{2}$, with transition temperatures reported to be as large as $200 \mathrm{~K}$ and $232 \mathrm{~K}$, respectively for bulk and monolayers [49]. The experimental evidence for the CDW phase was explained with an excitonic mechanism without invoking FS nesting and Jahn-Teller distorsion [50,51]. In contrast, DFT calculations show that the CDW phase in $\mathrm{TiSe}_{2}$ appears because of the stiffening of the shortranged Ti-Se force constant at a sufficiently low value [52]. Such stiffening manifests itself through the softening of the relevant phonon modes (yielding an enhancement of the electron-phonon coupling), which is here predicted for monolayer $\mathrm{TiS}_{2}$.

Intriguingly, we find that the formation of the CDW phase is not a unique feature of the $\mathrm{TiS}_{2}$ monolayer. In fact, we have repeated the phonon calculation for the $\mathrm{TiS}_{2}$ unit cell and a number of layers ranging from one to four and found imaginary phonon frequencies in all cases. In particular we report frequencies of $-92 \mathrm{~cm}^{-1},-52 \mathrm{~cm}^{-1},-18 \mathrm{~cm}^{-1}$ and $-21 \mathrm{~cm}^{-1}$, respectively for one, two, three and four layers (note that here we report the purely imaginary part of the frequency).
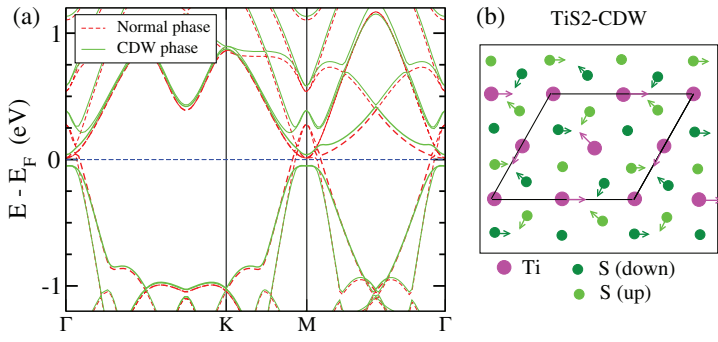

Fig. 4: (Color online) (a) The LDA bandstructure of a $\mathrm{TiS}_{2}$ monolayer $2 \times 2$ supercell in the normal (red dashed lines) and the commensurate CDW (green lines) phases. (b) The cartoon shows the commensurate CDW phase of the $\mathrm{TiS}_{2}$ monolayer (top view). The arrows indicate the planar displacement of the various atoms.

Considering that our accuracy over the frequency is of the order of $20 \mathrm{~cm}^{-1}$, we can conclude that the CDW phase may be present for both mono- and bi-layers and it vanishes for structures containing between three and four layers. This indicates that the interlayer interaction plays an important role in determining the condition for the CDW instability.

Another possibility for suppressing the CDW phase is by applying isotropic pressure, as already suggested for bulk $\mathrm{TiSe}_{2}$ [52]. This can be simulated by performing phonon bandstructure calculations for isotropically compressed unit cells and here we report results for the monolayer case. Importantly such compression does not alter significantly the electronic structure, and the material, as described by the LDA, remains metallic at all the pressures investigated with the only notable effect being an increase of the S $p$ bandwidth as the pressure gets larger. The situation, however, is different for the phonon bandstructure. As a demonstration in fig. 5(a) we present data for a compressive strain of $6.6 \%$. Interestingly, the minimum frequency of the soft-phonon mode at the $M$-point has an imaginary value $\left(-8 \mathrm{~cm}^{-1}\right)$ significantly smaller than the case when no pressure is applied $\left(-92 \mathrm{~cm}^{-1}\right)$. Consequently, the energy gain due to the CDW formation reduces with increasing the compressive strain. Eventually, the unstable mode disappears for a compressive strain of $7.1 \%$, which corresponds to a hydro-static pressure of $\approx 3.9 \mathrm{GPa}$. Such disappearance of the CDW can be explained by considering how the inter-atomic force constants change under compressive strain. Increasing the pressure causes the Ti-S bond length to decrease and enhances the stiffness of the nearest neighbors Ti-S force constant. As a consequence the energy of the soft-phonon mode increases and eventually the instability is removed. As such the CDW instability is sensitive on the local environment around the transition metal atoms in the $\mathrm{TiS}_{2}$ layer.

As mentioned before and demonstrated in fig. 4 the formation of the CDW is associated to a metal-to-insulator transition. One then expects the CDW phase to emerge 

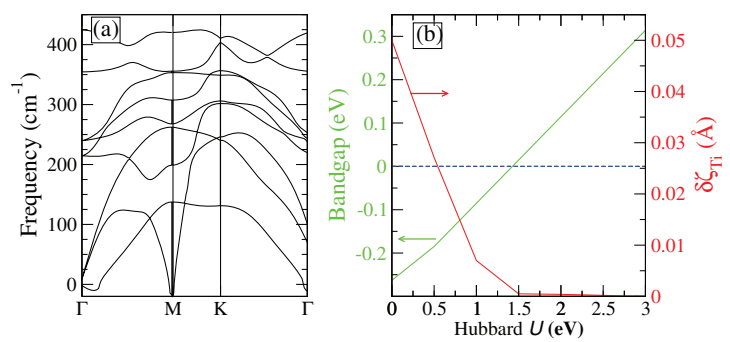

Fig. 5: (Color online) (a) Phonon dispersion of a $\mathrm{TiS}_{2}$ monolayer under a compressive strain of $6.6 \%$. (b) Variation of the $\mathrm{TiS}_{2}$ monolayer bandgap (green) of the undistorted phase and the Ti-distortion, $\delta \zeta_{T i}$ (red), against the Hubbard $U$ parameter for $\mathrm{LDA}+U$ calculations. A negative bandgap indicates a semimetallic ground state.

only in materials that are either metals or small-bandgap semiconductors in their undistorted geometry. The case of $\mathrm{TiS}_{2}$ is somehow unclear since there exists significant ambiguity on the experimental determination of the bandgap (or its absence). From the theoretical point of view it is important to mention that the LDA systematically underestimates the bandgap, so that the calculated semimetal ground state might not correspond to the correct one. To complicate the comparison with experiments there is also the fact that in LTMD monolayers the exciton binding energy can be as large as $1 \mathrm{eV}$, meaning that the quasiparticle and the optical gap may differ significantly from each other [53]. As such it is useful to revisit our results this time by using an exchange and correlation functional, which allows us to systematically open a bandgap in the undistorted phase. Considering the predominant $3 d$ character of the valence band, the $\mathrm{LDA}+U$ functional [38] appears to be an ideal choice, since the Coulomb repulsion $U$ parameter, can be used a control parameter for the opening of the gap.

In fig. 5(b) we report our results showing the variation of the bandgap of the undistorted phase and the Ti displacement, $\delta \zeta_{T i}$, as a function of $U$. Clearly we observe that the lattice distortion vanishes as soon as $U$ is large enough to open a bandgap in the undistorted phase. In other words the metallicity of the undistorted phase seems to be a necessary condition for the appearance of the CDW ground state. The next natural step is then to establish whether doping can produce the CDW ground state when the neutral system is described as an insulator. In order to explore this possibility we have run total energy calculations for the $2 \times 2$ supercell with either undistorted and distorted (CDW) geometries under different doping conditions. Doping is obtained by performing the calculation with a charged cell and a uniform compensating background. The LDA results are reported in fig. 6(a), while those obtained with $\mathrm{LDA}+U(U=4 \mathrm{eV})$ are in fig. 6(b).

From the figures it is clear that the LDA describes the possibility of a CDW for a relatively wide range of doping conditions, either $p$ or $n$. In contrast $\mathrm{LDA}+U$ favors

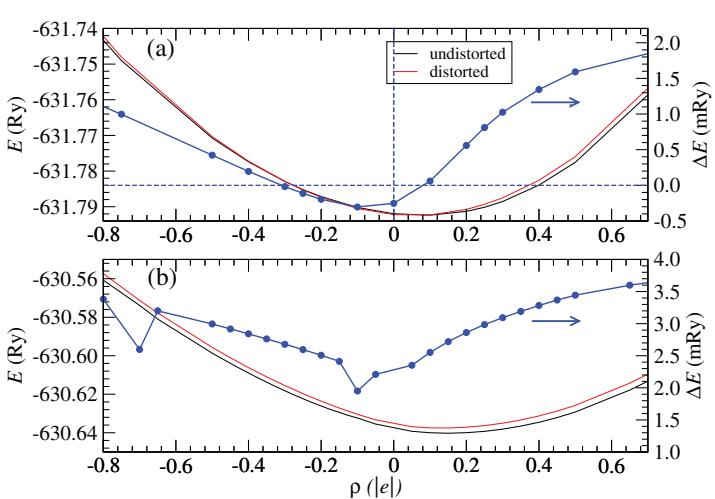

Fig. 6: (Color online) Total energy as a function of doping, $\rho$, for a $2 \times 2 \mathrm{TiS}_{2}$ supercell with either undistorted or distorted (CDW) geometry. On the right-hand side scale we report the energy difference, $\Delta E$, between the two phases, with negative values indicating CDW instability. Calculations are performed with (a) LDA and (b) $\mathrm{LDA}+U$.

the undistorted phases regardless of the level of doping. Notably the energy difference between the two phases are rather small in both cases. At this point we are not in the position to make a conclusive statement on the presence of the CDW. From our results it appears that this is found only when the calculation is performed at the LDA level and the undistorted ground state is semimetallic. Figure 6(b) shows that the distorted structure is not energetically favorable, which implies that doping a semiconducting bandstructure, as that obtained by $\mathrm{LDA}+U$, is not sufficient for the appearance of the CDW phase. As such we conclude that, although thinning $\mathrm{TiS}_{2}$ from bulk to a few layers significantly softens the phonon modes at the $\mathrm{M}$ point, this is enough to produce a CDW transition, which therefore remains only incipient in $\mathrm{TiS}_{2}$.

As mentioned above, the stiffness of the $\mathrm{Ti}-\mathrm{S}$ force constants plays a major role for an emergence of the CDW phase. This fact motivates us to study whether the CDW phase can be stabilized by tuning the stiffness, when the pristine monolayer is described as an insulator. This can be simulated by calculating, within the $\mathrm{LDA}+U$ (see footnote ${ }^{1}$ ), the phonon dispersion for isotropically strained unit cell and for the TiSSe alloy. The appearance of the CDW phase is always hindered by strain effects: a compressive strain monotonically increases the stiffness disfavouring the softening of the phonon modes, although the corresponding electronic bandgap reduces; similarly a tensile strain monotonically reduces the stiffness to the benefit of soft phonon modes but simultaneously increases the electronic bandgap. Therefore, the

\footnotetext{
${ }^{1}$ As the $\mathrm{LDA}+U$ is not implemented in the Quantum Espresso package for the phonon calculations, we have obtained results with the VASP [54] and PHONOPY packages [55]. As a test, we have also checked that a similar CDW phase, i.e. the appearance of the unstable phonon mode at the high symmetry $M$-point, can be reproduced within the LDA for the pristine $\mathrm{TiS}_{2}$ monolayer (see fig. 7(a)) by $V A S P$.
} 


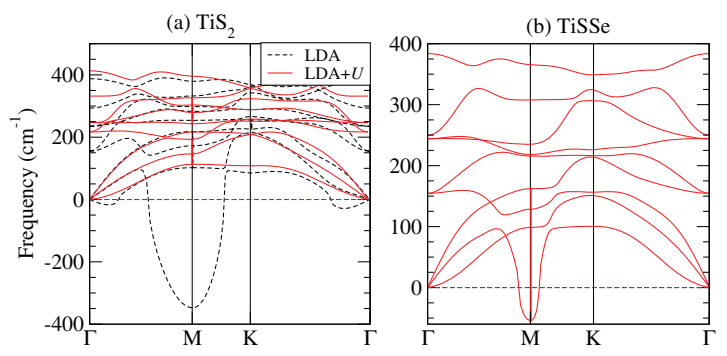

Fig. 7: (Color online) (a) The phonon dispersion of monolayer $\mathrm{TiS}_{2}$ calculated with the LDA (black dotted line) and the $\mathrm{LDA}+U$ (red line). (b) The phonon dispersion of the monolayer TiSSe alloy calculated with the $\mathrm{LDA}+U$. Note that the calculated imaginary frequencies are shown as negative roots of the square phonon frequencies.

enhancement of the stiffness is counterbalanced by the opening of the electronic bandgap. Note that metallicity in the undistored phase is required for the CDW phase transition. Indeed, we do not observe any imaginary phonon modes in the phonon dispersion of monolayer $\mathrm{TiS}_{2}$ for either compressive or tensile strain, i.e. strain alone cannot stabilize the CDW ground state. Next we calculate the phonon dispersion for the TiSSe alloy. This is constructed by replacing one $\mathrm{S}$ atom with one $\mathrm{Se}$ in the unit cell. Figure 7 shows the phonon dispersions of monolayer $\mathrm{TiS}_{2}$ pristine and of the TiSSe alloy, obtained with the $\mathrm{LDA}+U(U=4.0 \mathrm{eV})$. From the figures it is clear that the unstable phonon mode at the high symmetry $M$ point, an evidence of the CDW phase absent in pristine $\mathrm{TiS}_{2}$, can be retrieved by forming its alloy with Se. We have also checked that the TiSSe alloy exhibits the semimetallicity that is required for the presence of the CDW. Moreover, a Kohn anomaly of the phonon mode is observed in a $\mathrm{TiS}_{2-x} \mathrm{Se}_{x}$ alloy $(x \leq 37.5 \%)$, where the system has an insulating ground state. Note that here the alloy has been constructed from a $2 \times 2$ supercell of $\mathrm{TiS}_{2}$, in which $\mathrm{S}$ atoms are replaced with $\mathrm{Se}$ at the respective energetically favorable sites as suggested in ref. [13].

Conclusion. - In summary, we have performed electronic and phononic bandstructure calculations for $\mathrm{TiS}_{2}$ both in the bulk and monolayer form. In both cases the electronic structure derived from DFT-LDA is that of a semimetal with the band overlap points being different depending on the dimensionality. More interestingly we have found that the bulk form of $\mathrm{TiS}_{2}$ is undistorted, while significant phonon softening occurs at the $M$-point for nanostructures made of less than four monolayers. When one maintain the description of the electronic structure at the LDA level such softening yields a CDW ground state, which persists for a wide range of doping and can be strongly suppressed by compressive strain. However, if one adopts a description, such as the $\mathrm{LDA}+U$, where the electronic structure of the undistorted phase is insulating, such CDW state is no longer stable regardless of the doping. Since many-body quasi-particle theory suggests that $\mathrm{TiS}_{2}$ is insulating in its monolayer phase [42] we tentatively conclude that the CDW phase is only incipient in this material. However, alloying $\mathrm{TiS}_{2}$ with Se, for instance the formation of the TiSSe alloy, may give a viable option to bring back the CDW instability.

This work is supported by CRANN. We thank the Trinity Centre for High Performance Computing (TCHPC) for the computational resources provided.

\section{REFERENCES}

[1] Chen C. H., Fabian W., Brown F. C., Woo K. C., Davies B., Delong B. and Thompson A. H., Phys. Rev. B, 21 (1980) 615.

[2] Greenaway D. L. and Nitsche R., J. Phys. Chem. Solids, 26 (1965) 1445.

[3] Klipstein P. C. and Friend R. H., J. Phys. C: Solid State Phys., 17 (1984) 2713.

[4] Friend R. H., Jerome D., Liang W. Y., Mikkelsen C. and Yoffe A. D., J. Phys. C, 10 (1977) L705.

[5] Meakin J. I., Klipstein P. C. and Friend R. H., J. Phys. C, 20 (1987) 271.

[6] Thompson A. H., Pisharody K. R. and Koehler R. F., Phys. Rev. Lett., 29 (1972) 163.

[7] Lucovsky G., White R. M., Benda J. A. and Revelli J. F., Phys. Rev. B, 7 (1973) 3859.

[8] Fischer D. W., Phys. Rev. B, 8 (1973) 3576.

[9] Phillips J. C., Phys. Rev. Lett., 28 (1972) 1196.

[10] Fang C. M., de Groot R. A. and Haas C., Phys. Rev. $B, 56$ (1997) 4455.

[11] Liu B., Yang J., Han Y., Hu T., Ren W., Liu C., Ma Y. and Gao C., J. Appl. Phys., 109 (2011) 053717.

[12] YU Y. G. and Ross N. L., J. Phys.: Condens. Matter, 23 (2011) 055401.

[13] Cucinotta C. S., Dolui K., Pettersson H., Ramasse Q. M., Long E., OBrian S. E., Nicolosi V. and Sanvito S., J. Phys. Chem. C, 119 (2015) 15707.

[14] Friend R. H. and Yoffe A. D., Adv. Phys., 36 (1987) 1.

[15] Whittingham M. S., Science, 192 (1976) 1126.

[16] Inoue M., Hughes H. P. and Yoffe A. D., Adv. Phys., 38 (1989) 565.

[17] Benesh G. A., Woolley A. M. and Umrigar C., J. Phys. C: Solid State Phys., 18 (1985) 1595.

[18] Yu F., Sun J.-X. and Zhou Y.-H., Solid State Sci., 12 (2010) 1786

[19] Inosov D. S., Zabolotnyy V. B., Evtushinsky D. V., Kordyuk A. A., Büchner B., Follath R., Berger H. and Borisenko S. V., New J. Phys., 10 (2008) 125027.

[20] Johannes M. D. and Mazin I. I., Phys. Rev. B, 77 (2008) 165135.

[21] Liu A. Y., Phys. Rev. B, 79 (2009) 220515.

[22] Rossnagel K., J. Phys.: Condens. Matter, 23 (2011) 213001.

[23] Dai J., Calleja E., Alldredge J., Zhu X., Li L., Lu W., Sun Y., Wolf T., Berger H. and McElroy K., Phys. Rev. B, 89 (2014) 165140. 
[24] Rossnagel K., Kipp L. and Skibowski M., Phys. Rev. B, 65 (2002) 235101.

[25] Kidd T. E., Miller T., Chou M. Y. and Chiang T.-C., Phys. Rev. Lett., 88 (2002) 226402.

[26] Clerc F., Battaglia C., Cercellier H., Monney C., Berger H., Despont L., Garnier M. G. and Aebi P., J. Phys.: Condens. Matter, 19 (2007) 355002.

[27] Wang Q. H., Kalantar-Zadeh K., Kis A., Coleman J. N. and Strano M. S., Nat. Nanotechnol., 7 (2012) 699.

[28] Nicolosi V., Chhowalla M., Kanatzidis M. G., Strano M. S. and Coleman J. N., Science, 340 (2013) 1420.

[29] Splendiani A., Sun L., Zhang Y., Li T., Kim J., Chim C.-Y., Galli G. and Wang F., Nano Lett., 10 (2010) 1271.

[30] Goli P., Khan J., Wickramaratne D., Lake R. K. and Balandin A. A., Nano Lett., 12 (2012) 5941.

[31] Park K. H., Choi J., Kim H. J., Оh D.-H., Ahn J. R. and Son S. U., Small, 4 (2008) 945.

[32] Zeng Z., Tan C., Huang X., BaO S. and Zhang H., Energy Environ. Sci., 7 (2014) 797.

[33] Hohenberg P. and Kohn W., Phys. Rev., 136 (1964) B864.

[34] Kohn W. and Sham L. J., Phys. Rev., 140 (1965) A1133.

[35] Baroni S., de Gironcoli S., Dal Corso A. and Giannozzi P., Rev. Mod. Phys., 73 (2001) 515.

[36] Ceperley D. M. and Alder B. J., Phys. Rev. Lett., 45 (1980) 566.

[37] Giannozzi P. et al., J. Phys.: Condens. Matter, 21 (2009) 395502.

[38] Liechtenstein A. I., Anisimov V. I. and ZaAnen J., Phys. Rev. B, 52 (1995) R5467.

[39] Cococcioni M. and de Gironcoli S., Phys. Rev. B, 71 (2005) 035105.
[40] Allan D. R., Kelsey A. A., Clark S. J., Angel R. J. and Ackland G. J., Phys. Rev. B, 57 (1998) 5106.

[41] Wilson J. A. and Yoffe A. D., Adv. Phys., 18 (1969) 193.

[42] Zhu Z., Cheng Y. and Schwingenschlögl U., Phys. Rev. Lett., 110 (2013) 077202.

[43] Zeng Z., Yin Z., Huang X., Li H., He Q., Lu G., Boey F. and Zhang H., Angew. Chem., Int. Ed., 50 (2011) 11093.

[44] Ataca C., Şahin H. and Ciraci S., J. Phys. Chem. C, 116 (2012) 8983.

[45] Sandoval S. J., Chen X. K. and Irwin J. C., Phys. Rev. B, 45 (1992) 14347.

[46] Scharli M. and Lévy F., Phys. Rev. B, 33 (1986) 4317.

[47] Nishio Y., Shirai M., Suzuki N. and Motizuki K., J. Phys. Soc. Jpn., 63 (1994) 156.

[48] Wilson J. A., DiSalvo F. J. and Mahajan S., Phys. Rev. Lett., 32 (1975) 882.

[49] Chen P., Chan Y.-H., Fang X.-Y., Zhang Y., Chou M. Y., Mo S.-K., Hussain Z., Fedorov A.-V. and Chiang T.-C., Nat. Commun., 6 (2015) 8943.

[50] Hellmann S., Rohwer T., Kalläne M., Hanff K., Sohrt C., Stange A., Carr A., Murnane M. M., Kapteyn H. C., Kipp L., Bauer M. and Rossnagel K., Nat. Commun., 3 (2012) 1069.

[51] Sugawara K., Nakata Y., Shimizu R., Han P., Hitosugi T., Sato T. and Takahashi T., ACS Nano, 10 (2016) 1341.

[52] Calandra M. and Mauri F., Phys. Rev. Lett., 106 (2011) 196406.

[53] Komsa H.-P. and Krasheninnikov A. V., Phys. Rev. $B, 86$ (2012) 241201(R).

[54] Kresse G. and Furthmuller J., J. Comput. Mater. Sci., 6 (1996) 15.

[55] Togo A. and Tanaka I., Scr. Mater., 108 (2015) 1. 